\title{
Die Rezeption der deutschen Literatur in China vor dem Hintergrund der 4.-Mai- Bewegung am Beispiel der Übersetzungen von Guo Moruo
}

\section{Die 4.-Mai-Bewegung als Wendepunkt in der chinesischen Übersetzungsgeschichte}

Die 4.-Mai-Bewegung wird nicht nur als eine patriotische Studentenbewegung betrachtet, sondern im weiteren Sinne als eine geistige Erneuerungsbewegung verstanden, die sich zeitlich bis in die Mitte der 1920er Jahre erstreckt (vgl. Kuhn 2007, 188). Literargeschichtlich gesehen gilt sie sowohl als Beginn der modernen chinesischen Literatur als auch als ein wichtiger Wendepunkt in der chinesischen Übersetzungsgeschichte (vgl. Sun 1985, 55).

Vor der 4.-Mai-Bewegung legte man im Zuge der Verwestlichung beim Übersetzen den Schwerpunkt zunächst auf naturwissenschaftliche und technisch relevante Texte, um von der technischen Überlegenheit des Westens zu profitieren und die eigenen Schwächen zu beheben. Diese Hoffnung ging jedoch nicht in Erfüllung, und China blieb nach der Abdankung des letzten Kaisers der QingDynastie (1636-1912) und nach der Gründung der Republik im Jahr 1912 unter der Führung von Sun Yat-sen weiterhin ein rückständiges Land. Als Bestätigung dieser Einschätzung galt das diplomatische Scheitern in den Pariser Friedensverhandlungen. Man musste sich erneut über den Ausweg Chinas aus der Rückständigkeit Gedanken machen und nahm wieder einmal den Westen als Vorbild. Im Unterschied zu den vorigen auf den Fortschritt und auf die Stärkung Chinas abzielenden Versuchen, bei welchen die Chinesen von den westlichen Techniken sowie dem demokratischen politischen System lernen sollten, orientierten sich die Intellektuellen diesmal geistig am Westen. „Will man heutzutage die Politik erneuern, so muss man konsequenterweise auch die Literatur, die im Geist der Politiker nistet und herumspukt, erneuern“ (zitiert nach Yang 2000, 29), so schrieb Chen Duxiu, einer der wichtigsten Führer der Neue-Kultur-Bewegung. ${ }^{1}$ Zur Zeit der 4.-Mai-Bewegung

1 Chen Duxiu (1879-1942, geboren in der Provinz Anhui) war ein politischer Aktivist, Revolutionär und Anreger der Neue-Kultur-Bewegung der 1910er und 1920er Jahre, die auf die Schaf- 
begann man damit, ausländische Literatur mit bürgerlich-revolutionärem Ideengehalt in großem Umfang ins Chinesische zu übertragen und damit einem größeren Publikum vorzustellen - darunter auch bedeutende Werke der deutschen Literatur (vgl. Sun 1985, 55). Die übersetzten Texte entstammten verschiedenen Epochen und waren in unterschiedlichen Stilen verfasst. Die Entscheidung zur Auswahl der übersetzten Werke stand ,in sehr engem Zusammenhang mit der Entwicklungstendenz der chinesischen Gesellschaft sowie mit Mentalität und Neigung der Übersetzer“ (Sun 1985, 55). Zu dieser Zeit wurden Texte von Goethe, Schiller, Heine, Nietzsche, Hauptmann, Storm, Kleist und anderen dem chinesischen Publikum zugänglich gemacht, oftmals von bekannten Intellektuellen und Gelehrten ins Chinesische übersetzt. Sie erfreuten sich vor allem bei jungen Leuten großer Beliebtheit und leisteten einen großen Beitrag zur Modernisierung der chinesischen Literatur.

An dieser Stelle darf der Name Guo Moruo (1892-1978) nicht unerwähnt bleiben. Geboren in der südwestlichen Provinz Sichuan, wurde Guo einerseits in seiner Kindheit und Jugendzeit nach den Regeln der überlieferten konfuzianischen Lehre erzogen und kam andererseits infolge der Maßnahmen der Qing-Regierung zur Reform des traditionellen Bildungssystems mit neuer und westlicher Wissenschaft und Kultur in Berührung (vgl. Gong und Fang 1988, 6-7). Während seines Medizinstudiums in Japan erlernte er die Fremdsprache Deutsch, weil die japanischen Universitäten damals die deutsche medizinische Ausbildung kopierten. Die meisten Fremdsprachenlehrer Guos hatten ihr Studium an philologischen Fakultäten absolviert. Deshalb benutzten sie gerne literarische Texte als Lehrbücher, was Guo die Gelegenheit bot, die deutsche Literatur kennenzulernen (vgl. Huang et al. 2013, 29). Zur Zeit der 4.-Mai-Bewegung wirkte er nicht nur mit seinen zahlreichen Schriften an der kulturellen Erneuerung Chinas mit, sondern beteiligte sich auch als Übersetzer ausländischer Literatur aktiv daran. In Japan begann er seine Übersetzungsarbeit und verschaffte sich bald den Ruf eines kompetenten Übersetzers deutscher Literatur. Immensee von Theodor Storm, Goethes Die Leiden des jungen Werthers sowie Also sprach Zarathustra von Friedrich Nietzsche wurden in den 1920er Jahren von Guo Moruo nacheinander ins Chinesische übersetzt. Diese von ihm übertragenen Werke zählten zu den damals beliebtesten ausländischen Lektüren des chinesischen Publikums und wurden in kurzer Zeit mehrmals neu aufgelegt. Die genannten deutschen Autoren wurden

fung einer auf Demokratie und Wissenschaft basierenden neuen Kultur abzielte. Historisch gesehen galt die Neue-Kultur-Bewegung als die geistige Vorbereitung auf die darauffolgende 4.Mai-Bewegung, die umgekehrt als Höhepunkt und Fortsetzung der Neue-Kultur-Bewegung angesehen wurde. 
vielfach gelesen, öffentlich diskutiert und spielten im Modernisierungsprozess der chinesischen Literatur eine wichtige Rolle.

Im Folgenden wird zunächst ein Überblick über die chinesische Rezeption der deutschen Literatur in der Zeit der 4.-Mai-Bewegung am Beispiel der oben genannten Texte gegeben. Abschließend ist zu erläutern, wie sich die Rezeption ausländischer Literatur in den gesellschaftlichen und kulturellen Kontext Chinas einbettet. Denn es liegt „weniger am Text selbst, wie er wahrgenommen und gedeutet wird, als an seinen Lesern, ihren Interessen und Deutungsgewohnheiten. Rezeptionsgeschichten sind immer auch Geschichten der Entstellung und Abwandlung“ (Richter 2017, 21).

\section{Guos Übersetzungstätigkeit in der Zeit der 4.-Mai-Bewegung und ihre breite Rezeption}

Theodor Storm ist einer der bekanntesten deutschen Schriftsteller in China und seine Novelle Immensee gilt als sein beliebtestes Werk. Diese Novelle wurde sowohl auf dem Festland Chinas als auch in Hongkong und Taiwan mehrmals neu ins Chinesische übersetzt. Es sind mehr als 20 unterschiedliche Übersetzungen entstanden, was der Situation der Rezeption von Die Leiden des jungen Werthers in China ähnelt (vgl. Yang 2005, 166). Obwohl Auszüge aus Immensee bereits vor der 4.-Mai-Bewegung ins Chinesische übersetzt wurden, fand eine breite Rezeption der Novelle erst statt, als sie der Leserschaft in vollständiger Übersetzung vorlag. Diese bereitete Guo Moruo in Zusammenarbeit mit Qian Junxu $\mathrm{u}^{2}$ vor, doch wurde sie oft als Übersetzung von Guo allein angesehen, weil sie stark von Guos sprachlichem Stil geprägt ist (vgl. Yang 2005, 338). 1921 erschien die Übersetzung von Immensee im Verlag Taidong in Shanghai und wurde innerhalb von zehn Jahren allein in diesem Verlag vierzehnmal neu aufgelegt (vgl. Wei 2004, 108). Nur wenige Monate später publizierte The Commercial Press in Shanghai, das erste chinesische moderne Publikationsinstitut, eine weitere Übersetzung, angefertigt von Tang Xingtian. Diese Immensee-Ausgabe wurde von einer detaillierten Theodor-Storm-Biografie begleitet. Weil die zwei Übersetzungen zeitlich nah aufeinanderfolgten, war es nicht zu vermeiden, dass zwischen ihnen große Konkurrenz herrschte. Es entstand sogar eine polemische

2 Qian Junxu (1896-1994), auch als Qian Chao bekannt, war ein renommierter Medizinexperte. Während des Medizinstudiums in Japan hatten sich Qian und Guo kennengelernt. Um seine Deutschkenntnisse zu verbessern, begann Qian, Immensee ins Chinesische zu übersetzen. Guo Moruo überarbeitete Qians Manuskript, sowohl sprachlich als auch stilistisch. 
Debatte darüber, welche Übersetzung die bessere war. An dieser Auseinandersetzung nahm nicht nur Guo selbst teil, auch andere berühmte Gelehrte wie Ba Jin ${ }^{3}$ und Zheng Zhenduo ${ }^{4}$ beteiligten sich an der Diskussion. Im Jahr 1927 erschien schließlich eine dritte Übersetzung von Immensee, diesmal im Kaiming Shuju [Kaiming Verlag] in Shanghai. Zhu Xie, der Übersetzer dieser neuen Version, hielt es für sinnvoll, Storms Novelle erneut ins Chinesische zu übertragen, da die beiden vorherigen Übersetzungen Fehler enthielten und noch zu verbessern seien (vgl. Wei 2004, 110).

Dass drei chinesische Immensee-Übersetzungen innerhalb von nur sechs Jahren entstanden, zeigt, dass Storms Novelle zur damaligen Zeit äußerst populär war. Die darin beschriebene unerfüllte Liebe des jungen Paares Reinhard und Elisabeth fand große Resonanz bei vielen jungen Chinesen, die unter dem traditionellen Ehemodell, nämlich unter der von Familien und Eltern arrangierten Eheschließung, litten und sich nach selbstbestimmter Liebe sehnten (vgl. Yang 2005, 174). Zugleich waren die jungen Intellektuellen dieser Generation, die sich in der Konfrontation mit der Realität aus Enttäuschung oft einsam und melancholisch fühlten, von der empfindsamen Atmosphäre in Storms Darstellung fasziniert. Yu Dafu, ein bedeutender Schriftsteller jener Zeit, schrieb in der Vorrede zu Guo Moruos Immensee-Übersetzung:

Sobald wir seine [d. i. Theodor Storms] Erzählungen lesen, ist es immerhin nicht zu verhindern, in eine traurige Welt entführt zu werden. Wenn wir im Schein des Sonnenuntergangs am frühen Abend des Spätfrühlings oder Frühherbstes sein Immensee lesen, müssen wir uns verloren fühlen und es scheint so, als ob wir allmählich im dunklen Meeresboden versinken würden.

(Zitiert nach Wei 2004, 109)

Ein anderes Werk, das in dieser Zeit aus dem Deutschen ins Chinesische übersetzt und zu einer der beliebtesten Lektüren des chinesischen Publikums wurde, war Goethes Die Leiden des jungen Werthers. Die erste von den zahlreichen chinesischen Übersetzungen des Werthers stammte ebenfalls von Guo Moruo und sie wurde auch im Taidong Tushuju [Taidong Verlag] veröffentlicht, der ein Jahr zuvor Guos Immensee-Übersetzung publiziert hatte.

Zwar hatte Goethe schon vor der 4.-Mai-Bewegung den Weg nach China gefunden, doch verdankt er seine große Beliebtheit dieser besonderen Wendezeit.

3 Ba Jin (1904-2005, eigentlicher Name Li Yaotang, geboren in der Provinz Sichuan) war ein Schriftsteller, Übersetzer und Aktivist.

4 Zheng Zhenduo (1898-1958, geboren in der Provinz Zhejiang) war ein Schriftsteller, Lyriker, Übersetzer und Gelehrter.

5 Chinesischsprachige Titel und Zitate in diesem Beitrag wurden von der Verfasserin selbst ins Deutsche übersetzt. 
In der Anfangsphase der Goethe-Rezeption zog man oft einen Vergleich zwischen Goethe und Konfuzius - wie etwa der Gelehrte Ku Hung-Ming (1856-1928). In seinem um die Jahrhundertwende erschienenen Buch Aufzeichnungen eines Beraters aus der Residenz des Gouverneurs Zhang können wir nachlesen:

Ohne Eile, ohne Stagnation wie das Gestirn am Himmel wirken und die Tugend verbessern, um sich dem Ren [Güte, Humanität] anzunähern. Der hervorragende westliche Weise namens Ete [d. i. Goethe] ging in einer anderen Richtung zu demselben Ziel wie Konfuzius. In China wie in dem Abendland hat man den gleichen Weg. Wie lehrreich sein Spruch: sich immer strebend bemüht! Das Tao liegt also den Menschen nah, und in China wie auch in dem Abendland gibt es nur ein und dasselbe Tao. (Zitiert nach Yang 2005, 20)

Im Gegensatz zur konfuzianisch inspirierten Goethe-Rezeption der vorhergehenden Periode übernahm Goethe in der Zeit der 4.-Mai-Bewegung ,die neue Rolle eines Gewährsmannes des Emanzipationsversprechens der Moderne, in dessen Realisierung die Reform- und Revolutionsbewegung den einzig möglichen Weg zur nationalen Autonomie und Stärke Chinas erkennt“ (Jaeger 2011, 60). Man zog sogar eine Parallele zwischen der chinesischen Gegenwart der kulturellen Erneuerung und der Epoche des Sturm und Drang, weshalb großer Wert auf das Werk des jungen Goethe gelegt wurde. Im Jahr 1920 schrieb Guo Moruo an seine Freunde Tian Han und Zong Baihua und schlug ihnen vor, sich dem jungen Goethe zuzuwenden: „Ich denke, dass wir Goethes Werke so viel wie möglich vorstellen und erforschen müssen, da seine Zeit - die Zeit des ,Sturm und Drang` - unserer Zeit sehr ähnlich ist! Wir haben vieles von ihm zu lernen!“ (Zong et al. 2006 [1920], 18).

Dieser Brief und Dutzende weitere Briefe, die sich Guo, Tian und Zong vom 3. Januar bis zum 3. März 1920 schrieben, wurden in einen Band namens San Ye Ji [Kleeblatt] aufgenommen, der im Mai desselben Jahres im Shanghaier Taidong Verlag erschien. In seinem Vorwort erklärt Tian Han, dass sich ihre Korrespondenz hauptsächlich um einen Mittelpunkt drehte, nämlich um Goethe. Der oben erwähnte Vorschlag Guo Moruos fand die Zustimmung seiner Freunde. Jeder wählte für sich ein Thema aus, und Guo entschied sich für die Übersetzung von Faust und Die Leiden des jungen Werthers. ${ }^{6}$

6 Zong Baihua, Philosoph und Ästhetiker, war bereit, eine Abhandlung mit dem Titel „Zur Lebens- und Weltanschauung des deutschen Dichters Goethe“ zu verfassen; Tian Han, Dramatiker und Kritiker, wollte eine Goethe-Biografie sowie eine Abhandlung zur Beziehung von Goethe und Schiller schreiben, außerdem noch ein Kapitel von Shokamas „Forschungen über Goethes Gedichte“ aus dem Japanischen ins Chinesische übersetzen. Später wurden all diese Projekte mit Ausnahme von Tian Hans Goethe-Biografie in die Tat umgesetzt. Vgl. Yang 2005, 34. 
Mit der Veröffentlichung der chinesischen Werther-Übersetzung im Jahr 1922 erreichte die Goethe-Rezeption in China ihren ersten Höhepunkt: Goethe wurde ein Lieblingsautor der jungen Leute. Bereits im August 1923 erschien Guos Übersetzung in der vierten Auflage, ein Jahr später wurde sie zum achten Mal neu aufgelegt (vgl. Yang 2005, 40). In den folgenden Jahren brachten diverse Verlage weitere Neuübersetzungen des Werther auf den Markt. Der chinesische Goethe-Experte Yang Wuneng konstatiert treffend: „Das Büchlein schlug in China wie einst in Deutschland und Europa ein wie eine Bombe, so dass sein Verfasser Goethe beim chinesischen Publikum, insbesondere unter den Jugendlichen, über Nacht populär wurde“ (Yang 2005, 37). Es wurde sogar Mode, dass junge Liebespaare einander eine Werther-Übersetzung schenkten und sich das Versprechen gaben, dem Herzensbund ewig treu zu bleiben (vgl. Yang 2005, 39). Sie identifizierten sich mit der Werther-Figur und erkannten ihr eigenes Schicksal in der traurigen Geschichte wieder. Werthers emotionsgeladener Einbruch in das geregelte Familien- und Eheleben von Lotte und Albert empfanden sie wohl als ersehnten Protest gegen den Ahnen-, Familien- und Ehekult der konfuzianischen Überlieferung, in der das Konzept einer selbstbestimmten Liebe fast keine Bedeutung hat (vgl. Jaeger 2011, 61).

Wie die Rezeption von Goethe beginnt die von Friedrich Nietzsche ebenfalls vor der 4.-Mai-Bewegung. Zu Beginn des 20. Jahrhunderts wurde Nietzsche nicht als Philosoph, sondern als Dichter in China eingeführt und beeinflusste viele chinesische Schriftsteller tiefgreifend. So urteilte der Schriftsteller Mao Dun $^{7}$ über Nietzsche im Jahr 1920 beim Lesen von Also sprach Zarathustra wie folgt: „Nietzsche besitzt wirklich ein lyrisches Talent. Er ist mehr hervorragender Dichter als ausgezeichneter Philosoph“ (zitiert nach Wei 2004, 134-135).

Vor 1919 wurde der Name Nietzsche bereits von vielen führenden Intellektuellen - wie etwa Wang Guowei ${ }^{8}$, Lu Xun ${ }^{9}$, Chen Duxiu - mehrmals erwähnt, und sie alle schätzen Nietzsches Schriften zum „Übermenschen“ und zur „Umwertung aller Werte“. Als sich der Gelehrte und Schriftsteller Wang Guowei 1904 mit Nietzsches Philosophie auseinandersetzte, betonte er, dass dessen Lehre darauf abziele, „alte Kultur zu zerstören und neue Kultur zu schaffen“

7 Mao Dun (1896-1981, eigentlicher Name Shen Dehong) war ein berühmter Schriftsteller, Literaturkritiker und Aktivist.

8 Wang Guowei (1877-1927, geboren in der Provinz Zhejiang) war Schriftsteller, Lyriker und Gelehrter mit einem internationalen Ruf.

9 Lu Xun (1881-1936, eigentlicher Name Zhou Shuren) war Wegbereiter der modernen Literatur in China. Die im Jahr 1918 erschiene Kurzgeschichte Tagebuch eines Verrückten war der erste in Baihua (Umgangssprache) geschriebene Text in der Literaturgeschichte Chinas. 
(zitiert nach Yue 1980, 21). Nietzsche strebe nach einer Loslösung von der Belastung durch alte Traditionen und fordere den Umsturz aller Werte. Eine ähnliche Ansicht findet man auch im Text von Chen Duxiu unter dem Titel Aufruf an die Jugend aus dem Jahr 1915. Es handelt sich dabei um das Geleitwort des ersten Heftes der einflussreichen revolutionären Zeitschrift Neue Jugend, in der Chen die Gedanken Nietzsches als „Waffe“ gegen feudalistische Herrschaft nutzte. Auf Nietzsches Differenzierung zwischen Sklavenmoral und Herrenmoral zurückgreifend, bezeichnete Chen die in der chinesischen Tradition hochgeschätzte Moral also Treue, Gehorsamkeit, Anstand und Güte - als Sklavenmoral und stellte ihr die Herrenmoral entgegen, die vom Individuum ausgeht und Selbständigkeit und Gleichberechtigung in den Vordergrund rückt (vgl. Chen 2016a, 181-182). In seiner Schrift Der wahre Sinn des Lebens, die im Jahr 1918 in der Zeitschrift Neue Jugend erschien, betonte Chen Duxiu erneut, dass sich Nietzsche dafür einsetze, den individuellen Willen zu respektieren und eigenes Talent zu entfalten. Genau darin wollte Chen das Ziel des Lebens erkennen. Die „sogenannte Humanität“, Gerechtigkeit und Moral seien jedoch nichts als Betrügerei (vgl. Chen 2016b, 156). Ähnlich urteilten vor der 4.-Mai-Bewegung auch andere Intellektuelle über Nietzsches Lehre, beispielsweise Lu Xun, Cai Yuanpei ${ }^{10}$ und viele weitere - hier werden nicht alle aufgelistet. Ihren Stellungnahmen ist gemeinsam, dass sie eine besondere Aufmerksamkeit auf den rebellischen Geist von Nietzsches Philosophie richten und insbesondere Nietzsches Kritik der Überlieferung große Anerkennung zollen.

Die 4.-Mai-Bewegung setzte die Nietzsche-Rezeption fort und brachte deren ersten Höhepunkt in China hervor. Nach der Protestbewegung schrieb man nicht nur weitere Texte über den deutschen Philosophen, man beschäftigte sich auch intensiv damit, seine Schriften ins Chinesische zu übersetzen. Noch im selben Monat, in der die Studentenbewegung ausbrach, formulierte der Historiker $\mathrm{Fu}$ Sinian in der Zeitschrift Xinchao [The Renaissance] den Appell: „Wir müssen Laternen tragen und die Straßen entlang nach den Übermenschen suchen“ (zitiert nach Yue 1980, 21). Einige Monate später schrieb der Dramatiker Tian Han eine detaillierte Einführung zu Die Geburt der Tragödie im dritten Heft des ersten Bandes der Zeitschrift Shaonian Zhongguo [Jungchina] und unterstrich, dass man sich über die Leiden des Lebens hinwegsetzen und mit starkem Willen gegen sie ankämpfen müsse (vgl. Yue 1980, 21). In den folgenden Jahren bemühten sich Intellektuelle stetig darum, die chinesischen Leser mit Nietzsches Gedanken bekannt zu machen und sie vom darin enthaltenen

10 Cai Yuanpei (1868-1940, geboren in der Provinz Zhejiang) war Pädagoge und der erste Rektor der Peking Universität. 
rebellischen Geist zu überzeugen. In dieser Zeit unternahmen Intellektuelle wie Mao Dun, Lu Xun und Guo Moruo außerdem den Versuch, Nietzsches Werk Also sprach Zarathustra ins Chinesische zu übersetzen - die vergleichsweise vollständigste Übersetzung stammt von Guo Moruo. Von Mai 1923 bis Februar 1924 publizierte Guo nacheinander von ihm übersetzte Fragmente aus Zarathustra in der Zeitung Chuangzao Zhoubao [Schöpfungswochenzeitung]. Obwohl Guos Arbeit unvollendet blieb, wurde sie 1928 in Buchform veröffentlicht. Das Werk „wurde von der damaligen chinesischen Jugend mit Enthusiasmus begrüßt. Nietzsche wurde als Idolzerstörer gefeiert, der alle traditionellen Glaubensgrundsätze verworfen hat“ (Sun 1985, 57).

\section{Identifikationsversuch bei der Interpretation und in Einklang mit der Zeitstimmung}

Möchte man das Phänomen der Beliebtheit jener soeben erwähnten deutschen Werke bei den chinesischen Lesern begreifen, so ist es notwendig, ihre Rezeption im gesellschaftlichen, politischen und kulturellen Kontext jener Zeit zu betrachten. Was Goethe in seinem späten Leben zum Werther-Fieber in Deutschland sagt, trifft auch auf den unerwarteten Erfolg der oben erwähnten Texte im China der 1920er Jahre zu:

Die Wirkung dieses Büchleins war groß, ja ungeheuer, und vorzüglich deshalb, weil es genau in die rechte Zeit traf. Denn wie es nur eines geringen Zündkrauts bedarf, um eine gewaltige Mine zu entschleudern, so war auch die Explosion, welche sich hierauf im Publikum ereignete, deshalb so mächtig, weil die junge Welt sich schon selbst untergraben hatte, und die Erschütterung deswegen so groß, weil ein jeder mit seinen übertriebenen Forderungen, unbefriedigten Leidenschaften und eingebildeten Leiden zum Ausbruch kam.

(Goethe 1988, 580)

Die erwähnten Texte, die sich in den 1920er Jahren großer Beliebtheit bei dem chinesischen Publikum erfreuten, haben zur rechten Zeit ihren Weg nach China gefunden und den Zeitgeist getroffen. Zahlreiche Aspekte dieser Werke ließen sich auf die damals aktuellen Probleme Chinas übertragen, weshalb sie bei den Lesern große Resonanz fanden.

Bei der 4.-Mai-Bewegung handelte es sich nicht nur um eine patriotische Studentenbewegung, die gegen den Imperialismus kämpfte, sie war auch eine antifeudalistische Emanzipationsbewegung. Die Wendung gegen die imperiale Herrschaft verband sich mit einer Kritik an chinesischen Traditionen (vgl. Bauerkämper 2011, 21). Die in China über zweitausend Jahre lang herrschende Ethik 
der Selbstunterwerfung, die in den Drei Grundregeln und Fünf Grundbeziehungen des Konfuzianismus ihre Ausprägung fand, wurde nun von Grund auf in Frage gestellt. Von den Drei Grundregeln wurden die Beziehungen von Fürst zu Untertan, Vater zu Sohn und Ehemann zu Ehefrau geregelt, während die Fünf Grundbeziehungen das Verhältnis von Fürst zu Minister, Vater zu Sohn, älterem zu jüngerem Bruder, Ehemann zu Ehefrau und Freund zu Freund festlegten (vgl. Kuhn 2007, 192). Untertanen, Söhne und Ehefrauen wurden jeweils dem Fürsten, dem Vater und dem Ehemann untergeordnet und mussten ihnen gegenüber bedingungslosen Gehorsam leisten. Tugenden wie Humanität, Güte, Gerechtigkeit usw. zählten zu den wichtigsten Prinzipien, die man im Alltag verfolgen musste. Im alten China pflegte man nicht zu fragen, wer man ist, sondern welche Aufgabe man in der Gesellschaft und in der Familie übernehmen sollte (vgl. Hu 2011, 71-72). Das Individuum trat deshalb hinter der gesellschaftlichen und familiären Rolle zurück, was dazu führte, dass die individuelle Freiheit und Selbstständigkeit beeinträchtigt und vernachlässigt wurden. Zum Beispiel war im traditionellen Ehemodell keine freie Wahl des Ehepartners möglich, vielmehr wurde durch die Vermittlung der Eltern die Eheschließung arrangiert.

Die Vertreter der 4.-Mai-Bewegung versuchten, mit der Tradition zu brechen und gegen die für die konfuzianische Konvention typische Selbstunterwerfung zu kämpfen. Sie traten deshalb energisch für die Entfaltung der Persönlichkeit, für die individuelle Freiheit und nicht zuletzt für die Frauenemanzipation ein. Die Eheproblematik wurde $\mathrm{zu}$ einem aktuellen gesellschaftspolitischen Thema, und es fand sogar eine erste öffentliche „Liebesdebatte“ in China statt, an der bedeutende Intellektuelle - unter anderem Liang Qichao ${ }^{11}$ und Lu Xun - teilnahmen (vgl. Hu 2011, 67). Literarisch gesehen wurde das Ich zum zentralen Thema dieser Zeit; deshalb wurde die subjektive Wahrnehmung in den Vordergrund gerückt, was sich in den neuen Gattungen Tagebuch, Autobiographie und Ich-Erzählung niederschlug (vgl. Hu 2011, 65-66). Eben aus diesem Grund neigten die chinesischen Leser dazu, Nietzsches Forderung nach der „Umwertung aller Werte“ zuzustimmen, Werthers Aufstand gegen die Konvention auf die eigene Auseinandersetzung mit der konfuzianischen Überlieferung zu beziehen und sich mit der in Storms Novelle Immensee beschriebenen unerfüllten Liebe zwischen Reinhard und Elisabeth $\mathrm{zu}$ identifizieren. Die erwähnten Texte kamen zur richtigen Zeit in China an und passten bestens zur damals herrschenden Emanzipationsstimmung.

11 Liang Qichao (1873-1929) war ein Gelehrter, Philosoph und Reformer. Er zählte zu den führenden Kräften der gescheiterten Hundert-Tage-Reform der Qing-Dynastie. 
Wenn man die Rezeption der ausländischen Literatur verfolgt - besonders jene der hier vorgestellten Beispiele -, so fällt auf, dass die damaligen Leser dazu tendierten, die literarischen Inhalte in ihren eigenen Erwartungshorizont zu übertragen. Der subjektive und pragmatische Gesichtspunkt spielte bei der Interpretation der westlichen Literaturprodukte eine entscheidende Rolle, was oft dazu führte, dass die Geschichten nach den Bedürfnissen des Lesers vereinfacht ausgelegt wurden. Eben aus diesem Grund wurde Werther zum Sinnbild des Kampfes der jungen Leute gegen das traditionelle Ehemodell, und Reinhard und Elisabeth wurden als Opfer der von den Eltern nach feudalistischer Art bestimmten Eheschließung angesehen. Die Einfühlung in das Schicksal der Figuren trug einerseits zur breiten Rezeption dieser westlichen Literatur bei, anderseits behinderte sie ein umfassendes und differenziertes Verständnis der Werke. Zahlreiche Aspekte der Werke wurden vernachlässigt oder tauchten in der Diskussion erst gar nicht auf.

Zusammenfassend ist zu sagen, dass die 4.-Mai-Bewegung als kulturelle Erneuerung und geistige Neuorientierung in der neueren Geschichte Chinas den ersten Höhepunkt der Rezeption der ausländischen bzw. der deutschen Literatur mit sich bringt. Dank der Übersetzungstätigkeit von vielen Intellektuellen wie Guo Moruo sind zahlreiche ausländische klassische Texte dem chinesischen Publikum erstmals zugänglich geworden und haben zu der geistigen Emanzipationsbewegung Chinas einen großen Beitrag geleistet. Zugleich aber sollte man nicht außer Acht lassen, dass die ausländischen Werke durch die genannten Protagonisten der Emanzipationsbewegung in China eingeführt wurden und entsprechend instrumentalisiert und mit dem Zeitgeist in Einklang gebracht wurden. Ihre Rezeption ist tief in den zeitgenössischen gesellschaftlichen und kulturellen Kontext Chinas eingebettet.

\section{Literaturverzeichnis}

Bauerkämper, Arnd. „Kulturtransfer und Barrieren zwischen China und Deutschland in der Zwischenkriegszeit“. Deutsch-chinesische Annäherungen. Kultureller Austausch und gegenseitige Wahrnehmung in der Zwischenkriegszeit. Hrsg. Almut Hille, Gregor Streim und Pan Lu. Köln, Weimar und Wien 2011. 9-33.

Chen, Duxiu. „Jinggao Qingnian [Aufruf an die Jugend]“. Chen Duxiu Jingdian [Die klassischen Texte von Chen Duxiu: eine Auswahl]. Beijing 2016a. 180-185.

Chen, Duxiu. „Rensheng Zhenyi [Der wahre Sinn des Lebens]“. Chen Duxiu Jingdian [Die klassischen Texte von Chen Duxiu: eine Auswahl]. Beijing 2016b. 156-158.

Goethe, Johann Wolfgang. Werke. Hamburger Ausgabe, Band 9. München 1988.

Gong, Jimi, und Fang Rennian. Guo Moruo Zhuan [Guo Moruo. Eine Biografie]. Beijing 1988. 
Hu, Wei. „Die chinesische Rezeption von Goethes Dichtung und Wahrheit in den 1920er und 1930er Jahren“. Deutsch-chinesische Annäherungen. Kultureller Austausch und gegenseitige Wahrnehmung in der Zwischenkriegszeit. Hrsg. Almut Hille, Gregor Streim und Pan Lu. Köln, Weimar und Wien 2011. 65-75.

Huang, Manjun, Wang Zelong und Li Guoqian. Guo Moruo Zhuan [Guo Moruo. Eine Biografie]. Beijing 2013.

Jaeger, Michael. „Sturm und Drang in China. Goethe und die Vierte-Mai-Bewegung“. Deutschchinesische Annäherungen. Kultureller Austausch und gegenseitige Wahrnehmung in der Zwischenkriegszeit. Hrsg. Almut Hille, Gregor Streim und Pan Lu. Köln, Weimar und Wien 2011. 49-63.

Kuhn, Dieter. Die Republik China von 1912 bis 1937. Entwurf für eine politische Ereignisgeschichte. 3., überarbeitete und erweiterte Ausgabe. Heidelberg 2007.

Richter, Sandra. Eine Weltgeschichte der deutschsprachigen Literatur. München 2017.

Sun, Fengcheng. „Probleme der Wirkungsgeschichte neuerer deutschen Literatur in China“. Kontroversen, alte und neue. Akten des VII. internationalen Germanisten-Kongresses. Band 9. Hrsg. Albrecht Schöne. Göttingen 1985. 55-65.

Wei, Maoping. Deyu Wenxue Hanyishi Kaobian [Forschungen über die chinesische Übersetzungsgeschichte der deutschen Literatur]. Shanghai 2004.

Yang, Wuneng. San Ye Ji - Deyu Wenxue. Wenxue Fanyi. Bijiao Wenxue. [Kleeblatt - Deutsche Literatur. Literarische Übersetzung. Komparatistik]. Chengdu 2005.

Yang, Wuneng. Goethe in China (1889-1999). Frankfurt am Main 2000.

Yue, Daiyun. „Nicai Yu Zhongguo Xiandai Wenxue [Nietzsche und die moderne chinesische Literatur]“. Journal of Peking University (Humanities and Social Sciences) 3 (1980): 20-33.

Zong, Baihua, Tian Han und Guo Moruo. San Ye Ji [Kleeblatt]. Hefei 2006 [Erstausgabe Shanghai 1920]. 
